\title{
Gobernanza en el contexto contemporáneo: un análisis crítico de la perspectiva liberal
}

\author{
Governance in a contemporary context: a critical analysis of the liberal view
}

Daniel Javier de la Garza Montemayor ${ }^{1}$

Daniel Barredo Ibáñez ${ }^{2}$

\begin{abstract}
RESUMEN
Desde el último cuarto del siglo XX, se ha venido transformando el Estado, con la modernización de la administración pública y la democratización que incluye, entre sus principios básicos, una mayor participación ciudadana en el diseño, implementación y evaluación de las políticas públicas, dando lugar a la emergencia de la gobernanza. La gobernanza se define precisamente como el proceso de gobierno inclusivo que tiene como fundamento la participación de los diversos actores sociales, privados y públicos en las diferentes fases de planeación, implementación y evaluación de la administración pública y, más ampliamente, del gobierno. No obstante lo anterior, en la actualidad nos encontramos con que, en la práctica, la gobernanza contribuye a legitimar un modelo político y económico basado en los preceptos del neoliberalismo. Con el presente artículo, se busca contribuir a la discusión de los nuevos parámetros en los que se debaten las tendencias de la administración pública.
\end{abstract}

\section{Palabras clave}

Nueva gerencia pública, gobernanza, administración pública, Estado, neoliberalismo.

\begin{abstract}
Since the last quarter of XX century, the State has been changing with the modernization of public administration and the democratization, which includes as fundamental principles a greater citizen participation in the design, implementation and evaluation of public policies leading to the emergency of governance. Governance is just defined as the process of inclusive government founded on the participation of diverse social, private and public actors in the different stages of planning, implementation and evaluation of public administration and, more comprehensively, of the government. Nevertheless, at present we have found that, in practical terms, the governance contributes to legitimate a political and economic model based on the precepts of neoliberalism. This paper aimed to contribute to the review of new parameters in which the tendencies of public administration are discussed.
\end{abstract}

\section{Keywords}

New public management, governance, public administration, State, neoliberalism.

\section{Introducción}

En una obra trascendente que examina el estudio de la administración pública a través de la perspectiva de grandes teóricos de las Ciencias Sociales, Guerrero (2010) reconoce en John Stuart Mill a uno de los más importantes especialistas de la administración pública desde la perspectiva liberal.

En la visión de Mill, era importante que se estableciera una profesionalización (servicio

Doctor en Ciencias Políticas. Universidad Autónoma de Nuevo León (México).

Doctor en Periodismo. Universidad del Rosario (Colombia). 
civil) en el servicio público para separar esta función de la dinámica propia la política. Otro de los autores liberales que Guerrero (2010) destaca que es Herbert Spencer, para quien el fortalecimiento de la administración pública significaba el ascenso del socialismo.

Como consecuencia de la Revolución Rusa en 1917, y de la Gran Depresión a finales de los años 20 del siglo pasado, se comenzó a extender en el mundo capitalista la idea de revertir las condiciones de inequidad que caracterizaban al sistema económico y social con una mayor intervención estatal. Durante la presidencia de Frank Delano Roosevelt, se implementó en los EE.AA. el New Deal, que implicaba una fuerte intervención del Estado en la economía.

A partir de la Segunda Guerra Mundial, el mundo se divide en dos bloques que respaldaban sistemas contrapuestos. En este escenario, por una parte emerge la alternativa liberal a través de ideólogos que sostenían que el keynesianismo imperante en los países capitalistas resultaba improductivo, $y$ por el "socialismo real" que imperaba en las naciones del bloque soviético y que desde la visión neoliberal era el gran enemigo a vencer.

Existe una diferencia notable en cuanto al enfoque de los liberales clásicos respecto a los autores neoliberales. En este sentido, Guerrero (2004) establece la siguiente comparación:

en contraste con la economía clásica, especialmente con el pensamiento de John Stuart Mill, quien requirió el entendimiento de las leyes del desarrollo social, los austriacos se inclinaron por el conocimiento de los principios del desarrollo individual, conocimiento que sólo es posible observando a la persona actuando $y$ eligiendo en el mercado libre (p. 92).

Los autores a los que Guerrero (2004) hace mención son Ludwig von Mises y Friedrich Hayek, quienes tuvieron una enorme trascendencia en la construcción de los principios del neoliberalismo que sería implementado a partir de la década de los 70 . En el caso del primero, Guerrero (2010) hace referencia al convencimiento que tenía Von Mises en torno a que la economía y el Estado eran incompatibles.

En la perspectiva de Von Mises, la labor de la administración solo tenía sentido si estaba dirigida a salvaguardar principios como la vida y la libertad. A su vez, Von Mises consideraba que la burocracia no producía los resultados deseables en contraposición a los gastos que se generaban.

En cuanto al segundo autor, de acuerdo con Lessnoff (2011), Friedrich Hayek alcanzó su máximo grado de popularidad y auge durante el mandato de Margaret Thatcher. En la óptica de Hayek, la libertad era un valor supremo que estaba por encima de cualquier otro fin. De acuerdo con esta noción, un entorno que facilita que la sociedad persiga sus propios fines puede aspirar a ser grande.

Determinados principios que Hayek empleaba para la economía serían aplicados en el futuro a la Administración Pública. Entre ellos, el precepto de que la economía tenía una función materialista, en la que lo fundamental era que los individuos satisficieran sus aspiraciones. Esta concepción sería la base de una nueva Administración Pública en la que se concibe a los ciudadanos como "usuarios" que requieren servicios de calidad, en la misma lógica utilitarista que se examinará más adelante.

Resulta relevante mencionar que Lessnoff (2011) destaca que Hayek fue un detractor de los principios de la justicia social. Los principios de la economía se encuentran vacíos de cualquier elemento moral, por lo que son ajenos a cualquier principio de razón. Por otro lado, Lessnoff (2011) también indica que a Hayek no le simpatizaba una democracia de las mayorías, por lo que mostraba una predilección por un cuerpo legislativo que aprobara leyes que vigilaran un comportamiento íntegro en una colectividad.

Otro de los autores que influyeron en el enfoque neoliberal de la Administración Pública de acuerdo con Guerrero (2010) fue Walter Lippman, quien consideraba que un servidor público que interpretara la ley de 
acuerdo con su voluntad era la expresión de una visión absolutista.

En un concepto más amplio del "Estado Neoliberal", el entramado institucional se encuentra al servicio de dirimir conflictos entre individuos. De esta manera, la estructura estatal adquiere una dimensión procesal, en la que su función solo se justifica para garantizar el cumplimiento de las obligaciones que se han acordado entre particulares.

El neoliberalismo tuvo adherentes en el ámbito académico, así como de parte de algunos políticos después de la Segunda Guerra Mundial, pero se convirtió en una propuesta programática que sería aplicada hasta la década de los 70, como se mencionó de manera previa. Cabe destacar una publicación de Perlstein (2001), en la que hace la exposición de un estudio del programa electoral del candidato presidencial republicano en 1964, Barry Goldwater.

En la obra de Perlstein (2001), se narra cómo el contendiente republicano propuso una agenda "conservadora" que en muchos aspectos fundamentales contrastaba con las políticas sociales que enarbolaba su principal adversario, el entonces presidente Lyndon Johnson. Goldwater tuvo una derrota histórica en la elección, pero, en la perspectiva de Perlstein (2001), fue pionero en la elaboración de un programa que más tarde concretaría Ronald Reagan.

En la perspectiva de otros autores, la implementación del neoliberalismo distó de ser un evento fortuito. La primera experiencia de un gobierno que implementara políticas neoliberales fue en la década de los 70, bajo la dictadura de Pinochet en Chile. Años más tarde, Margaret Thatcher ganó las elecciones en el Reino Unido con una propuesta neoliberal. En relación al tema anterior, Monedero (2012) examinó el Informe a la Trilateral ${ }^{3}$ de 1975 , en el que se esboza un programa político que tendría repercusiones importantes en los años subsecuentes. En el texto, el autor indica que la
Trilateral establecía que uno de los problemas de las democracias de aquel entonces era que el Estado atendía diversas tareas, en parte por las crecientes demandas de la ciudadanía. Cabe recordar que, en buena medida, aún se encontraba presente en el imaginario colectivo la emergencia de movimientos sociales que sacudieron el panorama político a finales de la década de los 60. Por otra parte, el informe de la Trilateral identificaba como un problema, tanto al nacionalismo, como la ausencia de un dominio basado en liderazgos que tuvieran afinidad con la doctrina neoliberal.

En la percepción de Monedero (2012), el programa de la Trilateral:

se resume en: (1) menor participación popular en la política; (2) primacía de lo individual sobre lo colectivo; (3) sustitución del Estado por el mercado $y$ determinados actores sociales; (4) preponderancia del técnico sobre el político. Es el Management frente a la democracia (p. 306).

El modelo anterior se encontraba relacionado de manera intrínseca con un nuevo concepto de gobernabilidad. El autor concluye que lo anterior tuvo como consecuencia que predominara el discurso de la estabilidad frente al desarrollo social, mientras que en el camino quedaron anulados los principios de un Estado que buscara promover el bienestar general.

En una corta pero exhaustiva revisión de la historia del neoliberalismo reciente, los autores Steger y Roy (2010) analizan el fenómeno en función de su desarrollo. Clasifican el ascenso de Thatcher y Reagan como "la primera oleada del neoliberalismo". Los autores atribuyen a los gobernantes antes mencionados el haber logrado transmitir y aplicar de manera notable un mensaje en contra de los principios keynesianos que predominaban hasta entonces, mientras que defendían la necesidad de impedir la interferencia del Estado en el ámbito privado.

3 De acuerdo con el texto de Peterzell (1977), la Comisión Trilateral se fundó en 1973 por David Rockefeller y Zbigniew Brzezinski, con el fin de buscar la colaboración entre aquellos ciudadanos con una posición destacada que fueran cercanos a la toma de decisiones tanto en organizaciones públicas como privadas, de Norte América, Europa y Japón. 
No obstante lo anterior, los autores identifican una contradicción entre el discurso y la práctica. En la opinión de Steger y Roy (2010), la implementación del neoliberalismo fue posible gracias a la estructura del poder público. A partir de la utilización de una fuerza ideológica trasmitida a través de los organismos financieros internacionales, Thatcher y Reagan tuvieron éxito en imponer su agenda.

Los investigadores establecen que:

El mejor testimonio del poder que ostentaron el thatcherismo o la politica de Reagan lo tenemos en el hecho de que las fuerzas de la izquierda democrática pronto empezaron a incorporar a sus propios programas políticos las principales medidas del esquema neoliberal (p. 86).

Lo anterior resulta especialmente relevante debido a que durante la década de los 90 predominó un consenso en torno a que los programas de las diferentes alternativas políticas, independientemente de su signo ideológico, debían ajustarse a los principios del neoliberalismo. Este fue precisamente el auge del Consenso de Washington y el fin de la guerra fría simbolizado en la caída del muro de Berlín.

\section{Emerge la Gobernanza: ¿En detrimento del Estado?}

Analizaremos ahora el segundo constructo. El término gobernanza ha generado un modelo de lo que es considerado en diversos ámbitos como un ejercicio adecuado de gobierno, debido a la participación plural que implica. No obstante lo anterior, al igual que la Nueva Gestión Pública, existen autores que consideran que este concepto corresponde a una visión neoliberal del rol que debe asumir el Estado.

En una publicación crítica con el modelo de globalización predominante, Stiglitz (2002) advertía sobre la imposición de una visión de un Estado mínimo, y del predominio de los mercados en aquellos países que se encuentran en vías de desarrollo. En aquel entonces, proponía un cambio en la gobernanza, al democratizar las instituciones financieras como el Fondo Monetario Internacional y el Banco Mundial.

Una década más tarde, Stiglitz (2012) argumentaba que una de las salidas de la crisis económica generada a partir de 2008 era modificar la gobernanza de las grandes corporaciones, debido a que la legislación en Estados Unidos les permitía actuar de manera arbitraria e irresponsable.

En ambos casos, Stiglitz (2002, 2012) no propone una ruptura con la teoría de la gobernanza, debido a que considera que sus fundamentos pueden modificarse con el fin de generar las condiciones en los que se pueda contener los alcances de los poderes fácticos. En contraste, existen otras visiones que desacreditan esta noción en su conjunto.

En torno a lo anterior, Aguilar (2006), quien es uno de los principales proponentes del concepto de gobernanza, argumentaba que en la década pasada existían dos enfoques centrales en cuanto a la visión de una administración pública contemporánea. Una de ellas buscaba destacar el valor de lo público de la administración, mientras que la segunda iba orientada a renovar su capacidad administrativa que, según el autor "guarda indudablemente relación directa con las medidas de ajuste y equilibrio hacendario, que fueron indispensables para poder sacar al Estado desarrollador de su bancarrota fiscal" (p. 41).

En otra publicación, Aguilar (2007) argumenta que en el contexto actual: "el gobierno es un agente de dirección necesario pero insuficiente" (p. 6). En la perspectiva del autor, la emergencia de la gobernanza corresponde a una realidad en la que la actuación del gobierno resulta insuficiente para responder a los reclamos de la colectividad.

La presencia de la gobernanza corresponde a una evolución en la conducción de una comunidad, que permite la participación de nuevas entidades, de acuerdo con Aguilar (2007). En este caso, el autor destaca la necesidad de la colaboración entre la administración y la ciudadanía en el proceso de dirección a una sociedad. 
Por otra parte, una visión que resulta muy crítica con este concepto es la de Canto Sáenz (2012), quien establece que existe una equivocación al considerar que la participación política de los ciudadanos se asemeja a la gobernanza. Al respecto, Canto Sáenz (2012) argumenta que los ciudadanos pueden ejercer influencia en las acciones del gobierno, pero no asumen sus funciones. Por otra parte, en el término de gobernanza se considera que existen poderes que no han sido elegidos por la ciudadanía que también participan en la tarea de gobernar.

El autor distingue entre diferentes modalidades de la gobernanza, entre las que destaca una visión más moderada que promueve la participación de representantes del ámbito privado que asumen funciones que estaban reservadas para el Estado en el pasado. A pesar de que en esta versión se hace referencia a la necesidad de combatir las desigualdades sociales, aquellos que promueven una gobernanza democrática manifiestan su convicción de que estos problemas pueden atenuarse con la colaboración de particulares, sin la necesidad de realizar políticas que tenga un impacto en la redistribución de la riqueza.

Uno de los aspectos más críticos sobre el concepto de la gobernanza que Canto Sáenz (2012) distingue, es la ausencia de la acción política. Al delegar competencias de la administración pública a los particulares, el gobierno en su conjunto termina por disminuir su capacidad de hacer política. En la perspectiva del autor, el rol de la ciudadanía es clave en este proceso, debido a que tiene la responsabilidad de exigir un mejor funcionamiento del gobierno, así como una legislación acorde con sus demandas, en contraposición a permitir que estas responsabilidades sean delegadas.

En términos generales, Canto Sáenz (2012) coincide con Graña (2005), quien, años atrás, razonaba que la enorme mayoría de los teóricos de la gobernanza simpatizaban con la idea de un Estado mínimo. La postura de Graña es que el concepto de gobernanza coincide con un momento histórico en el que los lineamientos del Banco Mundial adquirieron una enorme relevancia en las últimas dos décadas del siglo pasado.

Tanto los gobiernos nacionales como las instituciones internacionales han propugnado por que los criterios a los que se ha hecho alusión, que mantienen una visión uniforme, sean aplicados en diferentes contextos que parten de realidades disímiles. Al respecto, Graña (2005) establece que: "todos los enfoques de la gobernanza planetaria comparten una convicción: Un estado debe intervenir menos y compartir su poder, debe costar menos y volverse más modesto" (p.518).

De acuerdo con Peters (2003), los agentes que participan en las decisiones generales que dan origen a la gobernanza no necesariamente son representativos del interés público. Al respecto, el autor advierte que existe el riesgo de generar un conflicto de interés en una situación en la que aquellos actores que inciden en determinadas decisiones fueran directamente los beneficiarios de las mismas.

En contraparte, Peters (2003) identificaba que en un caso contrario al antes expuesto se dificultaba también el funcionamiento de una gobernanza idónea. El autor expresaba que aun cuando las redes sociales estuvieran caracterizadas por la pluralidad e inclusión, plantear objetivos específicos que fueran legítimos para todas las expresiones sociales no se encontraba exento de impedimentos. A su vez, Peters (2003) distinguía que un modelo en el que existen tendencias tan disímiles, representaba un obstáculo para generar resoluciones.

A su vez, uno de los mayores riesgos dentro de la gobernanza que identifica Peters (2003), es que la toma de decisiones se concentrara en pocos participantes. Adicional a lo anterior, el autor destaca que en el esquema propuesto por la gobernanza, resulta complejo emplear la rendición de cuentas.

En un texto posterior, Peters (2004) identificaba que existían dos corrientes distintas que determinaron cambios en la perspectiva en la que se concebía a la administración pública. Por una parte, se partía de incorporar 
los métodos del mundo empresarial a la administración pública, mientras que existían otros que propugnaban por una nueva correspondencia entre el sector privado y los entes gubernamentales.

El enfoque antes descrito forma parte de la gobernanza que, según Peters (2004), lo coloca lejos del concepto original, "ahora se concibe al sector público como dependiente del privado de diversas maneras, y las políticas públicas se elaboran e implementan, en gran parte, mediante la interacción de los actores públicos y privados" (p. 72).

La pretensión de implantar principios universales en sociedades que son tanto complejas como divergentes supone, en primera instancia, un error de diagnóstico. En este caso, Trucco (2012) razona que el concepto de la gobernanza se encuentra en riesgo, mientras que a su vez considera que la interpretación que realizan los adherentes a esta teoría resulta discutible.

Existen otros elementos que, a juicio de Trucco (2012), obligan a reconsiderar los fundamentos de la gobernanza. Entre ellos se encuentra el hecho de que la gobernanza prescinde de un análisis histórico preciso, no considera las disparidades entre los elementos que formarían parte de ese gobierno colectivo, mientras que su interpretación de la sociedad tiene un componente más ideológico que metódico.

Por último, uno de los cuestionamientos más incisivos es el de Monedero (2009), quien califica a la doctrina de la gobernanza como una "trampa". En la opinión del autor, quienes pretenden imponer este concepto buscan suprimir los cuestionamientos críticos que provengan de una sociedad informada. El juicio de Monedero (2009) es contundente:

La gobernanza, en definitiva, se convierte en una forma de legalidad neoliberal, preparada incluso para ofrecer respuestas al colapso del neoliberalismo, ocultando una vez más con cuestiones de gestión la crítica de raíz al modelo capitalista. Es, como vimos al principio, una falsa síntesis que "opera totalmente dentro del marco de la gobernabilidad". Es de esta forma que los silencios de esta "matriz" son superiores a sus voces. Se calla la idea de transformación social, de una participación social no cooptada (los excluidos no están, aunque sí "sus representantes"), de justicia social, de poder, de conflicto, y por supuesto, de explotación y superación del marco capitalista. La idea de consenso, de cohesión, de coordinación y asociación, de autorregulación prepararon todo el marco que permitió la acumulación neoliberal de los ochenta y noventa y desembocó en la crisis económica del 2008 (p. 211).

Uno de los argumentos recurrentes que existe a favor de la implementación de la Gobernanza, es el hecho de que se parte del reconocimiento de una realidad en el que tanto el equilibrio como la colaboración entre las instituciones públicas y los actores sociales contribuyen a la democratización de un entorno determinado. En contraposición a la toma de decisiones de forma vertical y arbitraria, que tienden atribuirse a un Estado fuerte, en la gobernanza se pretende dar cauce a la colaboración de algunos grupos de la sociedad civil. A pesar de lo anterior, resulta posible argüir que en la práctica esta cooperación entre los grupos sociales y los representantes de los poderes públicos termina por diferir de los principios teóricos originales.

Como ejemplo de lo anterior, existe un reporte de Olivo Pérez, Alaníz Hernández, y Reyes García (2011), en el que se analiza la funcionalidad de los Consejos Escolares de Participación Social que es uno de los mecanismos que se han implementado en México con la finalidad de aumentar la calidad educativa.

En este espacio, convergen tanto la principal autoridad de una escuela, como el máximo representante de la Asociación de Padres de Familia, así como un testigo del Sindicato Nacional de Trabajadores de la Educación. La función de los Consejos Escolares de 
Participación Social ha sido motivo de cuestionamiento, debido a que su finalidad ha carecido de la suficiente precisión. Los autores argumentan que con base en las teorías de gobernabilidad, la finalidad de este encuentro sería en un sentido ideal la edificación de intercambios civilizados, pero en la práctica, esta interacción se realiza en base a un principio autoritario. Una de las principales metas es elevar la calidad del sistema educativo, que adquiere una complejidad notable al estar ausentes los elementos de transparencia y rendición de cuentas. Los autores cuestionan que los principios de gobernabilidad $y$ gobernanza no son aplicados de acuerdo con su propósito. La principal objeción es que la participación en el espacio de encuentro entre los tres actores se da a través de una articulación que los autores consideran forzada, no espontánea.

\section{Propuestas alternativas para la transformación de la Administración Pública}

Podría argumentarse que, las últimas dos décadas, los desacuerdos con el modelo neoliberal han tenido como respuesta propuestas que buscan moderar los aspectos que consideran más nocivos del sistema. La doctrina neoliberal y los preceptos que se han transferido a la administración pública son considerados por muchos autores como inevitables. A lo largo de este ensayo, se han registrado posturas de autores que en la actualidad son referencia en cuanto a las críticas que se han hecho al modelo; sin embargo, en un contexto adverso, sus planteamientos iban orientados a mitigar los excesos del sistema.

En este caso, cabe distinguir la obra de Uvalle (2003), quien desde la década pasada reivindicaba la relevancia de la administración pública en la democratización del Estado y en el bienestar colectivo. El autor ubica a la administración pública contemporánea en una estructura social $e$ institucional que resulta tanto compleja como diversa. Uvalle (2003) parte de la legitimidad de la administración pública como condición necesaria para afrontar con éxito los desafíos que se presentan en el contexto contemporáneo.

Con respecto a lo anterior, el autor destaca la importancia de la valoración que tiene la ciudadanía sobre la Administración Pública, así como la colaboración activa de las distintas expresiones sociales. También hace alusión al diseño procedimental que permite la rendición de cuentas, el mismo que se traduce en un funcionamiento más eficaz del Estado. La principal diferenciación de Uvalle (2003) frente a los autores que argumentan a favor de un modelo de Administración Pública basado en los principios de la Nueva Gestión Pública, es que el autor mantiene un énfasis especial en la ampliación del espacio público.

Enlaperspectiva de Uvalle (2012), lagobernanza moderna se encuentra intrínsecamente ligada a los méritos democráticos. En contraste con el argumento que esgrimen determinados autores en torno a la deficiencia del Estado como uno de los orígenes de la gobernanza, Uvalle (2012) atribuye a la administración pública un rol preponderante en la gobernanza democrática. Resulta pertinente destacar que una de las características que según Uvalle Berrones (2012) distinguen a la gobernanza democrática es: "el vigor de lo público" (p. 120).

En consecuencia con lo anterior, es importante distinguir que Uvalle (2003) habla sobre el público usuario, que se refiere a la relación clave entre la administración pública y la ciudadanía. Lo anterior se encuentra basado en resultados concretos que dan legitimidad a este vínculo. A su vez, se hace referencia a la rendición de cuentas, así como a la evaluación pública, temas interrelacionados que resultan de gran trascendencia en una administración pública participativa. Lo anterior se debe a que resulta posible definir las responsabilidades de la administración, así como los indicadores que se establecen para su valoración.

En términos generales, resulta posible argüir que la principal divergencia de Uvalle (2003) con un enfoque neoliberal es que, mientras ambos enfoques en el discurso solicitan la participación de la ciudadanía en los asuntos públicos, en el primer caso la importancia 
de la administración pública no es materia de debate, al contrario, es un componente fundamental (Uvalle: 2011).

En cuanto al caso de la gobernanza, Uvalle argumenta que la administración debe concentrar los esfuerzos tanto de las entidades económicas como sociales, y en priorizar los temas de interés colectivo. Una de las principales consecuencias de materializar pactos entre los diferentes entes sociales, de acuerdo con el autor, es alcanzar el bienestar común. A su vez, Uvalle (2011) identifica que lo anterior se traduce en generar la aceptación de la colectividad al sistema vigente, su legitimación.

Por otro lado, también es importante destacar que Uvalle (2003) considera que el Estado debe ser el principal garante de los derechos humanos, libertades cívicas y del bienestar general, en comparación con los autores con orientación neoliberal quienes expresan que el Estado debe limitar su papel a ser un ente procesal.

En el caso de una obra posterior, Uvalle (2007) establece algunos aspectos que resultan relevantes para reivindicar el rol de la administración pública en un régimen democrático. En esta obra, distingue el espíritu de lo público, así como los aspectos y valores que conciernen a la ciudadanía como es el caso de la vida colectiva, la ética y la confianza.

En consecuencia con lo anterior, Uvalle (2007) señala la trascendencia del espacio público en las sociedades plurales, así como la institucionalización del ejercicio del poder. En contraposición al ideario que propugna por la disminución del Estado, Uvalle (2007) insiste sobre la importancia de lo público en un entorno con alta participación de los ciudadanos, condición que considera necesaria para materializar avances en la justicia social.

\section{Conclusiones}

A lo largo de este artículo, se han documentado algunos de los principios del pensamiento neoliberal que funcionaron como inspiración en la elaboración de conceptos que marcaron una visión que pretendía ser unánime sobre la administración pública.

Elauge del concepto de gobernanza difícilmente puede disociarse de un proceso crucial que inició en los años 70. No obstante lo anterior, al entrar el modelo económico en crisis, también surgieron fuertes cuestionamientos a los modelos de administración pública que se encontraban de manera intrínseca relacionados con ese proyecto.

En este espacio, se ha argumentado también con base en investigaciones previas, que el precepto de gobernanza tiene un fuerte componente ideológico, más que bases científicas que sustenten sus planteamientos. Por otra parte, en la práctica, la implementación de ambos constructos no ha sido experiencia del todo exitosa, por lo menos hasta ahora. 


\section{REFERENCIAS}

Aguilar, L. (2006) Gobernanza y gestión pública. Fondo de Cultura Económica: México.

Aguilar, L. (2007) El aporte de la política pública y de la nueva gestión pública a la gobernanza, en Reforma y Democracia, Vol. 50, 1-5.

Canto Sáenz, R. (2012) Gobernanza y democracia. De vuelta al río turbio de la política, en Gestión y Política Pública, Vol. 21, N. ${ }^{\circ}$ 2, 333-374.

Espino Sánchez, G. (2014) 2011, Año de Gracia. A manera de presentación, en G. Espino Sánchez (Coord.), La insatisfacción con la democracia en México. Política convencional, movimientos sociales y tecnologías digitales. México: Fontamara. 7-15.

Gómez Díaz de León, C. (1998) Administración pública contemporánea. McGraw-Hill: México.

Graña, F. (2005) Todos contra el Estado: Usos y abusos de la "gobernanza", en Espacio Abierto, Vol. 14, N. ${ }^{\circ} 4,501-529$.

Guerrero Orozco, O. (2004) La nueva gerencia pública: Neoliberalismo en la Administración Pública. Fontamara: México.

Guerrero Orozco, O. (2010) La administración pública a través de las ciencias sociales. Fondo de la Cultura Económica: México.

Lessnoff, M. H. (2011) La filosofía política del siglo XX. Akal: Madrid.

Monedero, J.C (2012) El programa de máximos del neoliberalismo: el Informe a la Trilateral de 1975, en Sociología Histórica, Vol. 1, 289-310.

Monedero, J.C. (2009) El gobierno de las palabras. Política para tiempos de confusión. Fondo de la Cultura Económica: México.

Olivo Pérez, M. Alaníz Hernández, C. y Reyes García, L. (2011) Crítica a los conceptos de gobernabilidad y gobernanza. Una discusión con referencia a los consejos escolares de participación social en México, en Revista Mexicana de Investigación Educativa, Vol. 16, N. ${ }^{\circ}$ 50, 775-799.

Perlstein, R. (2001) Before the Storm. Barry Goldwater and the Unmaking of the American Consensus. Nation Books: Estados Unidos.

Peters, G. (2003) La capacidad para gobernar: ¿rretrocediendo hacia el centro?, en Reforma y Democracia, Vol. 27, 1-15.

Peters, G. (2004) Cambios en la naturaleza de la administración pública: De las preguntas sencillas a las respuestas difíciles, en Pardo, C. (Comp.), De la Administración Pública a la Gobernanza. México: Colegio de México. 69-101.

Steger, M.B y Roy, R.K. (2010) Neoliberalismo. Una breve introducción. Alianza Editorial: España.

Stiglitz, J. (2002) El malestar en la globalización. Santillana: México. 
Stiglitz, J. (2012) El Precio de la desigualdad. El 1 por ciento de la población tiene lo que el 99 por ciento necesita. Taurus: México.

Uvalle Berrones, R. (2003) Instituto de Administración Pública del Estado de México, A.C: México.

Uvalle Berrones, R. (2007) Perspectiva de la administración pública. Instituto de Administración Pública del Estado de México, A.C: México.

Uvalle Berrones, R. (2011) Las políticas públicas en el arquetipo de la gobernanza democrática. Reforma y democracia, Vol., N. ${ }^{\circ}$ 50, 1-13.

Uvalle Berrones, R. (2012) La administración pública en los imperativos de la gobernanza democrática. Convergencia, Vol. 19, N. ${ }^{\circ}$ 60, 111-144.

Fecha de recepción: 05-09-17

Fecha de aceptación: 08-10-17 